\title{
Thin-layer Chromatography of Cyclodextrins
}

\author{
By Ken'ichi TAKEo, Yôtaro Kondo* and Takashi Kuge \\ Department of Agricultural Chemistry \\ Kyoto Prefectural University, Kyoto \\ Received October 27, 1969
}

The cyclodextrins are a series of homologous cyclic oligosaccharides obtained from starch by the action of Bacillus macerans amylase. In the dextrins $\alpha-, \beta$-, and $\gamma$-dextrins are respectively composed of six, seven and eight $D$ glucopyranoses that are linked at $\alpha, 1 \rightarrow 4$ in a cyclic form. The dextrin exhibit a unique character to form inclusion compounds with a wide variety of organic and inorganic compounds, in which a single dextrin molecule provides hollow space available for the inclusion of guest molecules.

In the course of the studies on the inclusion complexes of the dextrins by means of gas chromatography, ${ }^{1 \prime}$ polarography ${ }^{2 \prime}$ and X-ray diffractions, ${ }^{3,4}$ a simple and rapid procedure has been required to characterize the dextrins produced during the $B$. macerans enzymolysis of starch and to check the purity of the individual dextrins.

Several chromatographic techniques have been described for the separation of dextrins. Cramer et al. ${ }^{5,6)}$ succeeded in separating $\alpha$-,

* Present address: Department of Agricultural Chemistry, Kyoto University, Kyoto.

1) T. Kuge and K. Takeo, Agr. Biol. Chem., 32, 753 (1968).

2) T. Kuge and K. Takeo, Abstracts of Papers for the Annual Meeting of the Agricultural Chemical Society of Japan (in Japanese), Nagoya, April, 1968, p. 25.

3) K. Takeo and T. Kuge, Agr. Biol. Chem., 33, 1174 (1969).

4) K. Takeo and T. Kuge, Abstracts of Papers for the Annual Meeting of the Agricultural Chemical Society of Japan (in Japanese), Tokyo, April, 1969, p. 66 .

5) F. Cramer and D. Steinle, Ann., 595, 81 (1955). $\beta$-, and $\gamma$-dextrins by circular paper chromatography. This method usually requires an irrigation period of about $20 \sim 25 \mathrm{hr}$ to permit their isolation. On the other hand, Wiedenhof $^{71}$ reported the separation of dextrins by using the technique of microchromatoplate developed by Peifer. ${ }^{8 !}$ However, in this case, no description has been given for the separation of $\gamma$-dextrin.

Because of the long separation time in paper chromatography and of the lack of the method for the separation of $r$-dextrin in microchromatoplate, both methods do not seem to satisfy our requirements. Accordingly, conventional thin-layer chromatography was examined for efficiency in separating dextrins. The thin-layers examined were Kieselguhr (Kieselguhr G, Merck), microcrystalline cellulose (Avicel SF, Funakoshi Chem. Co. Ltd.) and silicagel (Kieselgel G, Merck). All the plates were prepared in the usual manner to give a thickness of $250 \mu$. Both the plates of Kieselguhr and silicagel were allowed to stand at room temperature for $1 \mathrm{hr}$, and placed in an oven at $110^{\circ} \mathrm{C}$ for $30 \mathrm{~min}$ and then stored over silicagel in a dessicator. The plates of cellulose were dried overnight in the air and used without further conditioning. The following solvent systems which are commonly used for the separation of linear oligosaccharides were examined in various proportions, i.e., n-butyl alcohol-

6) F. Cramer and F. M. Henglein, Chem. Ber., 91, 308 (1958).

7) N. Wiedenhof, J. Chromatog., 15, 100 (1964).

8) J.J. Peifer, Mikrochim. Acta, 352, 529 (1962). 
ethyl alcohol-water, $n$-butyl alcohol-pyridinewater, $n$-butyl alcohol-glacial acetic acid-water and $n$-butyl alcohol-isopropyl alcohol-water, etc. Three $\sim$ five $\mu \mathrm{g}$ of a pure dextrin or about $20 \mu \mathrm{g}$ of a mixture of the dextrins were applied to a streak on the plates and the plates were irrigated in an ascending manner in a glass vessel with fresh solvent without prior equilibrium. The irrigation was continued until the front of the solvent ascended about $10 \sim 13 \mathrm{~cm}$ from the original point. After the completion of the irrigation, the plates were dried on a hot plate and detection was made by spraying $1 \%$ methanolic iodine solution. Distinct colors developed instantaneously, that is, violet for $\alpha$-dextrin, yellow for $\beta$ dextrin and brown for $\gamma$-dextrin. This method made simultaneous detection of the individual dextrins possible in a short time.

After trying various combinations of the thin-layers and the solvent systems mentioned above, the following two combinations were found to give good results in separating dextrins; (I) microcrystalline cellulose layer with $n$-butyl alcohol-ethyl alcohol-water

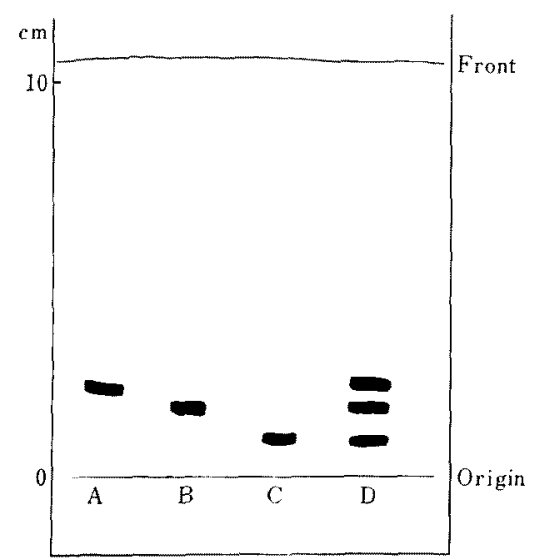

FIG. 1. Thin-layer Chromatogram of Cyclodextrins on Microcrystalline Cellulose.

Solvent system, n-butyl alcohol-ethyl alcoholwater ( $4: 3: 3$ by volume); (A) $\alpha$-cyclodextrin, (B) $\beta$-cyclodextrin, (C) $\gamma$-cyclodextrin, (D) mixture of $\alpha-, \beta$, and $\gamma$-cyclodextrins.
( $4: 3: 3$ by volume) and (II) Kieselguhr layer with $n$-butyl alcohol-ethyl alcohol-water $(4: 3: 2$ by volume). When the combination (I) was

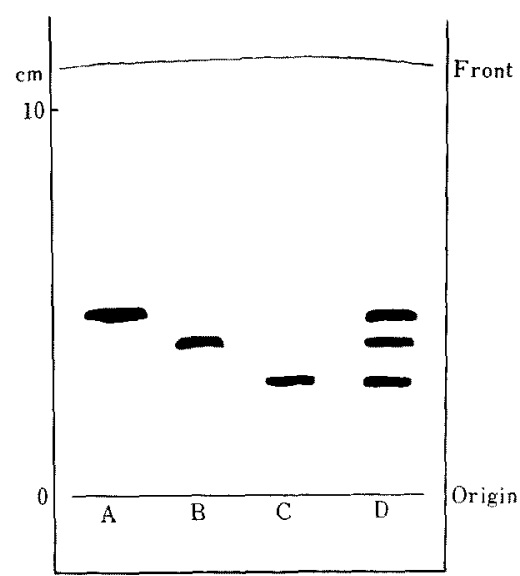

FIG. 2. Thin-layer Chromatogram of Cyclodextrins on Microcrystalline Cellulose Obtained after Twicerepeated Irrigations.

Solvent system, n-butyl alcohol-ethyl alcoholwater ( $4: 3: 3$ by volume); (A) $\alpha$-cyclodextrin, (B) $\beta$-cyclodextrin, (C) $\gamma$-cyclodextrin, (D) mixture of $\alpha^{-}, \beta-$, and $\gamma$-cyclodextrins.

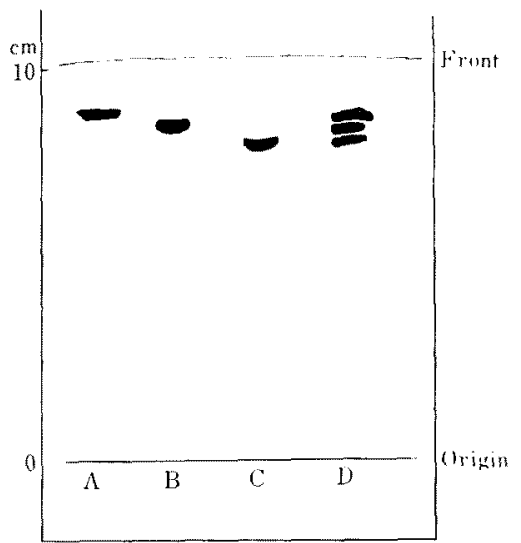

FIG. 3. Thin-layer Chromatogram of Cyclodextrins on Kieselguhr $\mathrm{G}$.

Solvent system, $n$-butyl alcohol-ethyl alcoholwater ( $4: 3: 2$ by volume); (A) $\alpha$-cyclodextrin, (B) $\beta$-cyclodextrin, (C) $\gamma$-cyclodextrin, (D) mixture of $\alpha^{-}, \beta-$, and $\gamma$-cyclodextrins. 
used, the results obtained were satisfactory for the separation of the dextrins as shown in Fig. 1. The individual dextrins were resolved in sharply defined bands without tailing. The time of the irrigation was about $4 \mathrm{hr}$. Irrigations repeated twice with the same mixture of the solvents by a multiple ascending technique gave better resolution as shown in Fig. 2. Thus, the $R f$ values considerably increased. When the combination (II) was used, irrigation was completed in a short time of about $1.5 \mathrm{hr}$. The chromatogram obtained is shown in Fig. 3. In this case, separation was rather poor and the spots were less sharp than when the combination (I) was used. However, each dextrin can obviously be distinguished by inherent color formation with iodine spray. In both the cases of (I) and (II), there is no interference for the detection of the dextrins with linear oligosaccharides which may also be produced during $B$. macerans enzymolysis, since the oligosaccharides only form a very faint color with iodine.

Attempts to separate the dextrins by the use of silicagel were unsuccessful due to appreciable tailings of the spots regardless of solvent systems.

The above experimental results now demonstrate the usefulness of thin-layer chromatography for the separation and identification of the dextrins. The present procedure has the advantage of sharp resolution as well as speed as compared with paper chromatography. It may be said that the combination (I) is most suitable for the checking of the purity of the individual dextrins, and that the combination (II) may be helpful for a quick check of the appearance of the dextrins during the enzymolysis of starch by $B$. macerans amylase. 